University of Wollongong

Research Online

Faculty of Engineering and Information

Faculty of Engineering and Information

Sciences - Papers: Part A

Sciences

$1-1-2016$

Statistical bias and variance for the regularized inverse problem: application to space-based atmospheric $\mathrm{CO} 2$ retrievals

Noel A. Cressie

University of Wollongong, ncressie@uow.edu.au

R Wang

Ohio State University

Michael M. Smyth

Jet Propulsion Laboratory NASA

Charles E. Miller

Jet Propulsion Laboratory NASA

Follow this and additional works at: https://ro.uow.edu.au/eispapers

Part of the Engineering Commons, and the Science and Technology Studies Commons

Research Online is the open access institutional repository for the University of Wollongong. For further information contact the UOW Library: research-pubs@uow.edu.au 


\title{
Statistical bias and variance for the regularized inverse problem: application to space-based atmospheric $\mathrm{CO} 2$ retrievals
}

\author{
Abstract \\ Remote sensing of the atmosphere is typically achieved through measurements that are high-resolution \\ radiance spectra. In this article, our goal is to characterize the first-moment and second-moment \\ properties of the errors obtained when solving the regularized inverse problem associated with space- \\ based atmospheric $\mathrm{CO} 2$ retrievals, specifically for the dry air mole fraction of $\mathrm{CO} 2$ in a column of the \\ atmosphere. The problem of estimating (or retrieving) state variables is usually ill posed, leading to a \\ solution based on regularization that is often called Optimal Estimation (OE). The difference between the \\ estimated state and the true state is defined to be the retrieval error; error analysis for OE uses a linear \\ approximation to the forward model, resulting in a calculation where the first moment of the retrieval error \\ (the bias) is identically zero. This is inherently unrealistic and not seen in real or simulated retrievals. \\ Nonzero bias is expected since the forward model of radiative transfer is strongly nonlinear in the \\ atmospheric state. In this article, we extend and improve OE's error analysis based on a first-order, \\ multivariate Taylor series expansion, by inducing the second-order terms in the expansion. Specifically, we \\ approximate the bias through the second derivative of the forward model, which results in a formula \\ involving the Hessian array. We propose a stable estimate of it, from which we obtain a second-order \\ expression for the bias and the mean square prediction error of the retrieval.
}

\section{Keywords}

variance, regularized, inverse, problem, bias, application, statistical, space, atmospheric, co2, retrievals

\section{Disciplines}

Engineering | Science and Technology Studies

\section{Publication Details}

Cressie, N., Wang, R., Smyth, M. \& Miller, C. E. (2016). Statistical bias and variance for the regularized inverse problem: application to space-based atmospheric CO2 retrievals. Journal of Geophysical Research: Atmospheres, 121 (10), 5526-5537. 


\section{Journal of Geophysical Research: Atmospheres}

\section{RESEARCH ARTICLE \\ 10.1002/2015JD024353 \\ Key Points: \\ - The retrieval of $X \mathrm{CO}_{2}$ is a nonlinear \\ Statistical bias and variance for the regularized inverse problem: Application to space-based atmospheric $\mathrm{CO}_{2}$ retrievals}

ill-posed inverse problem with

nonzero bias

- First-moment and second-moment statistical properties of atmospheric $\mathrm{CO}_{2}$ retrievals from satellite remote sensing instruments are approximated

- The approximations are assessed in a realistic simulation experiment and found to perform well

Correspondence to:

N. Cressie,

ncressie@uow.edu.au

Citation:

Cressie N., R. Wang, M. Smyth, and C. E. Miller (2016), Statistical bias and variance for the reg ularized inverse problem: Application to space-based atmospheric $\mathrm{CO}_{2}$ retrievals, J. Geophys. Res. Atmos., 121, 5526-5537, doi:10.1002/2015JD024353.

Received 15 OCT 2015 Accepted 13 APR 2016 Accepted article online 3 MAY 2016 Published online 27 MAY 2016

O2016. American Geophysical Union. All Rights Reserved.

\author{
N. Cressie ${ }^{1,2}$, R. Wang ${ }^{3}$, M. Smyth ${ }^{4}$, and C. E. Miller ${ }^{4}$ \\ ${ }^{1}$ National Institute for Applied Statistics Research Australia, University of Wollongong, Wollongong, New South Wales, \\ Australia, ${ }^{2}$ Jet Propulsion Laboratory, Pasadena, California, USA, ${ }^{3}$ Department of Statistics, Ohio State University, \\ Columbus, Ohio, USA, ${ }^{4}$ Jet Propulsion Laboratory, NASA, Pasadena, California, USA
}

Abstract Remote sensing of the atmosphere is typically achieved through measurements that are high-resolution radiance spectra. In this article, our goal is to characterize the first-moment and second-moment properties of the errors obtained when solving the regularized inverse problem associated with space-based atmospheric $\mathrm{CO}_{2}$ retrievals, specifically for the dry air mole fraction of $\mathrm{CO}_{2}$ in a column of the atmosphere. The problem of estimating (or retrieving) state variables is usually ill posed, leading to a solution based on regularization that is often called Optimal Estimation (OE). The difference between the estimated state and the true state is defined to be the retrieval error; error analysis for OE uses a linear approximation to the forward model, resulting in a calculation where the first moment of the retrieval error (the bias) is identically zero. This is inherently unrealistic and not seen in real or simulated retrievals. Nonzero bias is expected since the forward model of radiative transfer is strongly nonlinear in the atmospheric state. In this article, we extend and improve OE's error analysis based on a first-order, multivariate Taylor series expansion, by inducing the second-order terms in the expansion. Specifically, we approximate the bias through the second derivative of the forward model, which results in a formula involving the Hessian array. We propose a stable estimate of it, from which we obtain a second-order expression for the bias and the mean square prediction error of the retrieval.

\section{Introduction}

Remote sensing of the atmosphere by satellites is typically achieved through a combination of physics and statistics. The physics is captured through a forward function, yet every retrieval is recognized to have error associated with it. This article presents a statistical approach to estimating the mean and variance of the retrieval error (the retrieved state vector minus the true state vector) from radiances that are connected to the state through a forward function that is modeled using a simpler forward model. Our goal here is retrieval of atmospheric $\mathrm{CO}_{2}$, and we work within the framework of Rodgers [2000], using a nonlinear forward model and Bayesian inverse methods; this has sometimes been called Optimal Estimation (OE).

The error analysis given by Rodgers [2000] is a first-order analysis that proceeds as if the forward model is linear, and it always gives the result that the mean of the retrieval is equal to the prior mean. Assuming this model is correct, the bias (i.e., the mean of the retrieval error) is zero. However, because the forward model is nonlinear, in reality there is a nonzero bias that we henceforth call nonlinearity bias.

In this article, we develop expressions that quantify the nonlinear contributions to atmospheric $\mathrm{CO}_{2}$ retrieval errors; they are based on capturing nonlinearity through the Hessian array, which is the second derivative of the forward model. Its stable estimation is critical, and a key contribution of this article is to present a weighted estimate that preserves symmetry properties of the Hessian array. Our results apply to any retrieval obtained by solving an inverse problem through regularization, and hence they are applicable to the many remote sensing retrievals that are based on a radiative transfer function.

In the context of remote sensing of atmospheric $\mathrm{CO}_{2}$, there have been a number of articles discussing $\mathrm{OE}$ of the state based on instruments such as the Greenhouse Gases Observing Satellite (GOSAT) and OCO-2 [see, e.g., Böesch et al., 2006; Connor et al., 2008; Kuze et al., 2009; Bréon and Ciais, 2010; Böesch et al., 2011; Crisp et al., 2012; O'Dell et al., 2012; Cressie and Wang, 2013]. In this article, we develop statistical methodol- 
ogy for a second-order error analysis that recognizes nonlinearity of the forward model connecting the state of the atmosphere with high-resolution radiance spectra measured by the remote sensing instruments on board GOSAT and OCO-2 [e.g., Crisp et al., 2014]. The full multivariate distribution could be obtained through a Markov chain Monte Carlo (MCMC) algorithm, but the computing time per retrieval for models of this complexity takes on the order of a day for 250,000 iterations (running a single MCMC chain per CPU on a Jet Propulsion Laboratory (JPL) 64 bit Linux cluster at approximately $2.8 \mathrm{GHz}$ (J. Hobbs, personal communication, 2016)). Algorithms of this type are presented clearly in Tamminen and Kyrola [2001] and Tamminen [2004], albeit for different instrumental and atmospheric configurations.

In section 2, we present details of the experiment we conducted to look at the properties of the retrieval error, including bias due to nonlinearity of the forward model. Section 3 briefly reviews OE and its error analysis. Section 4 presents our statistical methodology that recognizes and estimates bias due to the inherent nonlinearity of the forward model. This is done through a second-order, multivariate Taylor series expansion resulting in the Hessian array; using the approach of statistical estimating equations, a stable estimate of the Hessian array is derived. In section 5, we present the results of the experiment described in section 2. Discussion and conclusions are presented in section 6 .

\section{Synthetic OCO-2 Data: A Controlled Experiment}

This section describes an experiment we conducted to determine the influence of nonlinearity on the mean and variance of the retrieval error. More details are given in section 5, where the results of the experiment are presented. The experiment involves simulations from a known nonlinear forward model, where the true atmospheric state is known (but not used in the retrieval).

Radiances are simulated that are typical of those seen by GOSAT, with an ACOS forward model [Crisp et al., 2012] that emulates retrievals from OCO-2: The atmospheric state is first simulated; then the nonlinear forward model is applied (here version B5.0 of the ACOS/OCO-2 forward model); and finally, measurement error is added to yield synthetic high-resolution radiance spectra. Critically, because in the experiment the state is known, the retrieval error can be obtained exactly. From a large number of such simulations, the distribution of the retrieval error can be obtained. In this article, our goal is to obtain estimates of the dry air mole fraction of $\mathrm{CO}_{2}$ in a column $\left(\mathrm{XCO}_{2}\right)$, which is the key quantity used in flux inversion, along with its uncertainty quantification.

Repeating this under different scenarios, most notably location/season (varying albedo, aerosol optical depth, and vertical distributions), "aerosols," and "clear sky," generates scenario-specific distributions for the retrieval errors. Recall that within the experiment, the mean of the retrieval error is simply the bias due to nonlinearity of the forward model; that bias must be zero if the forward model is linear and the modeling assumptions given above hold.

Based on a pilot study to determine the important geophysical factors to consider, the presence/absence of aerosols emerged as the one that was most sensitive to nonlinearity bias. Hence, we chose 18 GOSAT locations over Australia during different seasons in order to generate a variety of atmospheres (see Figure 1), and we simulated with aerosols present or not. The mean of the state (i.e., the prior mean) was obtained from climatology, as is done for OCO-2 retrievals [Crisp et al., 2014], and the prior covariances were chosen so that physically meaningful atmospheres were generated by the simulation. Using a multivariate Gaussian distribution, we simulated 700 random atmospheric states for each scenario (i.e., for each given location/ season and each given presence/absence of aerosols). Relative Monte Carlo accuracy to the first decimal place is achieved with this number of simulations.

For each simulated state, a radiance vector was simulated, again by generating from version B5.0 of ACOS/OCO-2's full-physics forward model [Crisp et al., 2014] and random noise was added to capture errors due to measurement by the instrument on board the satellite. The end result was that for each scenario, 700 synthetic radiance vectors were available for analysis.

For each radiance vector, the next step in the experiment was to retrieve the atmospheric state, particularly the column-averaged dry air mole fraction $\left(\mathrm{XCO}_{2}\right)$ in parts per million $(\mathrm{ppm})$. To exercise strong control on the experiment, we use exactly the same forward model, measurement-error covariance, prior mean, and prior covariance for the retrieval as were used in the simulations. The robustness of the retrievals and the associated error analysis to misspecifications of these components is not addressed here; our goal is to work within the 


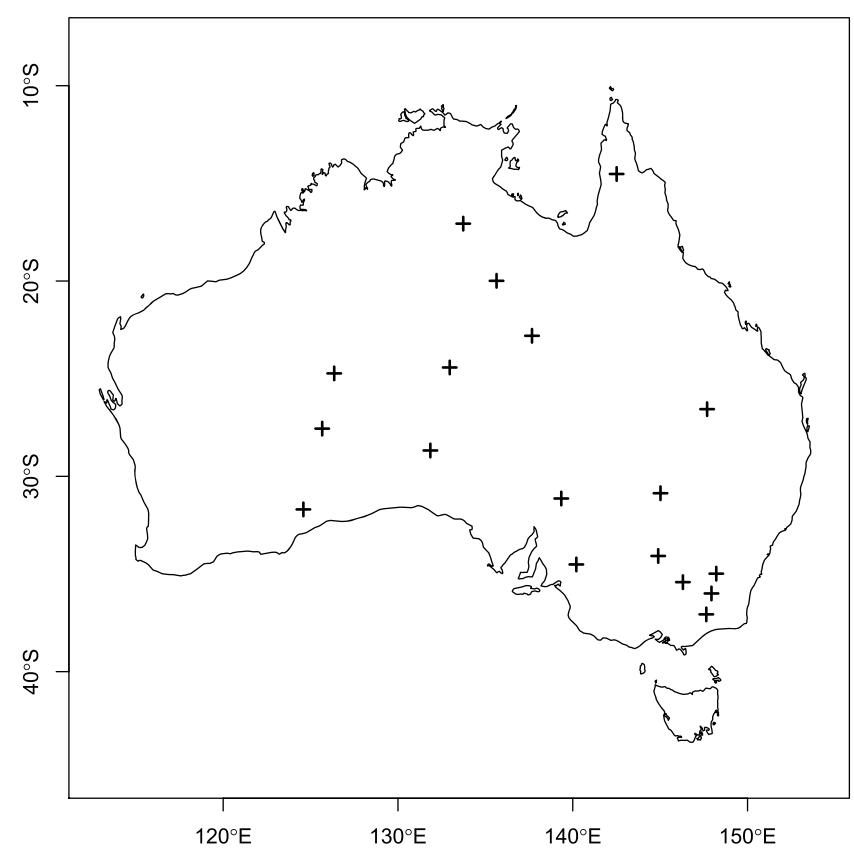

Figure 1. Map showing the 18 locations at which retrievals were performed during different seasons. At each location, 700 atmospheres and radiances were simulated using climatology, a full-physics forward model, and known measurement-error characteristics.

assumptions of $\mathrm{OE}$ in order to isolate the effect due to nonlinearity of the forward model. That is, we avoid confounding errors due to model misspecification with errors due to nonlinearity.

Consequently, for each of the 700 radiance vectors, there is an estimated state vector obtained from the level 2 algorithm, B5.0. Although not used in the retrieval, we also have available the respective true state vectors, from which we can trivially construct, by subtraction, 700 retrieval error vectors (= estimated state vector-true state vector). In practice, the true state vector is never known, and one must use statistical methodology to obtain properties (e.g., first and second moments, or all moments through MCMC) of the retrieval error distribution. Hence, the distribution of retrieval error for each scenario, obtained from the simulation, represents the gold standard against which all error analyses, linear and nonlinear, can be compared.

In the next section, we establish the necessary notation to discuss the state space model and the retrieval algorithm obtained from it. Finding the first-moment and second-moment statistical properties of the retrieval error of the multivariate state is the focus of this article; section 5 gives the results obtained from the controlled experiment described above.

\section{Radiative Transfer Function and Optimal Estimation}

In this section, we describe briefly the statistical model given in Rodgers [2000], and we summarize the specific implementation used for the ACOS/OCO-2 retrievals. The first part of the statistical model is based on the physics of radiative transfer that links measured radiances to the physical state of a column of the atmosphere sampled by the light path. It is here that the measurement uncertainty is accounted for, and in the terminology of state space estimation, the first part is called the measurement equation.

The second part of the statistical model recognizes that the atmospheric state vector (e.g., $\mathrm{CO}_{2}$ volume mixing ratios at different pressure levels, surface pressure, aerosols, albedo, and so forth) is not completely certain, and its uncertainty is described by a statistical distribution with a given mean vector and a given covariance matrix. In the terminology of $\mathrm{OE}$, these are called the prior mean vector and the prior covariance matrix, respectively. In the terminology of state space estimation, these are called the mean and covariance of the state, respectively, and the second part is called the state equation; see, for example, Shumway and Stoffer [2006], for a description of state space modeling and estimation. Finally, the measurement equation and the state equation are used in Bayes Theorem to obtain the posterior distribution, upon which the ACOS/OCO-2 retrieval is based. 


\subsection{State Space Model}

As explained above, the statistical model for retrieving the atmospheric state (x) from measured radiances (y) is divided into two parts. The measurement equation, which includes the (typically nonlinear) forward model $\mathbf{F}(\mathbf{x})$, is

$$
\mathbf{y}=\mathbf{F}(\mathbf{x})+\varepsilon
$$

In (1), $\varepsilon$ is an $n_{\varepsilon}$-dimensional error vector that captures both measurement error in the radiances $\mathbf{y}$ and specification error incurred by approximating the physics of radiative transfer through a generally nonlinear "working function" (or forward model) $\mathbf{F}($ ). For the remote sensing application considered in section 5, $n_{\varepsilon}=2040$. Assume that $\varepsilon \sim \operatorname{Dist}\left(\mathbf{0}, \mathbf{S}_{\varepsilon}\right)$, where $\mathbf{S}_{\varepsilon} \equiv \operatorname{cov}(\varepsilon)$ is a given $n_{\varepsilon} \times n_{\varepsilon}$ covariance matrix of measurement errors and $\operatorname{Dist}(\boldsymbol{\mu}, \boldsymbol{\Sigma})$ denotes a generic multivariate distribution with mean vector $\boldsymbol{\mu}$ and covariance matrix $\Sigma$; there may be other parameters that determine the distribution, but our interest centers on the first two. For example, Dist is often chosen to be the multivariate Gaussian (Gau) distribution. Cressie and Wang [2013] consider the general case where $E(\varepsilon) \neq \mathbf{0}$, and they show how the data $\mathbf{y}$ can be corrected to account for a nonzero mean. Hence, we can assume without loss of generality that $E(\varepsilon)=\mathbf{0}$.

The state $\mathbf{x}$ is an $n_{\alpha}$-dimensional vector (typically, $n_{\alpha} \ll n_{\varepsilon}$ ), and it is not known exactly; the state equation (called the prior model, in the terminology of $\mathrm{OE}$ ) expresses this uncertainty through

$$
\mathbf{x}=\mathbf{x}_{\alpha}+\alpha
$$

where $\mathbf{x}_{\alpha}$ is a known prior mean specification of the state, $\boldsymbol{\alpha}$ is an error vector, and all vectors in (2) are $n_{\alpha}$-dimensional. It is assumed that $\boldsymbol{\alpha} \sim \operatorname{Dist}\left(\mathbf{0}, \mathbf{S}_{\alpha}\right)$, where $\mathbf{S}_{\alpha} \equiv \operatorname{cov}(\boldsymbol{\alpha})$ is a given $n_{\alpha} \times n_{\alpha}$ covariance matrix, and note that Dist for $\alpha$ may be different from that for $\varepsilon$ in (1). For the remote sensing application considered in section $5, n_{\alpha}=50$.

We note that there remain parameters in the forward model and variance-covariance parameters in (1) and (2) that need to be specified or estimated. A fully Bayesian approach would put prior/hyperprior distributions on them; in this article, we assume that parameters of the measurement and state equations are completely known, as does Rodgers [2000] and subsequent articles on OE.

The inverse problem is to infer the state $\mathbf{x}$ from data $\mathbf{y}$; this results in the estimated state $\hat{\mathbf{x}}$, which in remote sensing is typically used to compute geophysical parameters of the atmosphere (e.g., $\left.X \mathrm{CO}_{2}\right)$. The ACOS/OCO-2 algorithm uses Twomey-Tikhonov regularization [Tikhonov, 1963; Twomey, 1963] to solve the problem; see section 3.2 .

Let $\left\{x(p): 0 \leq p \leq P_{\text {su }}\right\}$ denote the $\mathrm{CO}_{2}$ volume mixing ratios (VMRs) for every pressure level $p$ from the surface pressure $P_{\mathrm{su}}$ to the top of the atmosphere. Then, for predetermined pressure levels, $0 \leq p_{1}<p_{2}<\cdots<p_{n_{p}} \leq P_{\text {su' }}$ we define the $n_{p}$-dimensional subvector,

$$
\mathbf{x}_{p} \equiv\left[\begin{array}{c}
x\left(p_{1}\right) \\
\vdots \\
x\left(p_{n_{P}}\right)
\end{array}\right]
$$

We then partition the full state vector $\mathbf{x}$ into two (or possibly more) components as

$$
\mathbf{x} \equiv\left[\begin{array}{l}
\mathbf{x}_{p} \\
\mathbf{x}_{Q}
\end{array}\right],
$$

where $\mathbf{x}_{p}$ is that part of the state vector pertaining to the $\mathrm{CO}_{2}$ profile and $\mathbf{x}_{Q}$ represents all non- $\mathrm{CO}_{2}$ state vector elements, including the surface pressure, surface albedo, profiles of $\mathrm{H}_{2} \mathrm{O}$, temperature, and aerosols. Using obvious notation, we see that the dimension of $\mathbf{x}_{Q}$ is $n_{Q}=n_{\alpha}-n_{p}$. Hence, we can partition the prior mean as

$$
\mathbf{x}_{\alpha} \equiv\left[\begin{array}{c}
\mathbf{x}_{P, \alpha} \\
\mathbf{x}_{Q, \alpha}
\end{array}\right]
$$


and the prior covariance matrix as

$$
\mathbf{S}_{\alpha} \equiv\left[\begin{array}{ll}
\mathbf{S}_{P P, \alpha} & \mathbf{S}_{P Q, \alpha} \\
\mathbf{S}_{P Q, \alpha}^{\prime} & \mathbf{S}_{Q Q, \alpha}
\end{array}\right] .
$$

As will be seen in section 5 , it is useful to have the definitions (3)-(6), in order to extract statistical properties of estimated $\mathrm{CO}_{2}$ values from the statistical properties (given in section 4) of the full state vector $\mathbf{x}$.

\subsection{The Retrieval}

Rodgers [2000] proposes an OE (often called a Bayesian) solution to the retrieval problem, which we summarize below. The maximum a posteriori estimator is defined to be the state (or set of states, if it is not unique) $\hat{\mathbf{x}}$ such that the posterior distribution evaluated at $\hat{\mathbf{x}}$ satisfies

$$
P(\hat{\mathbf{x}} \mid \mathbf{y}) \geq P(\mathbf{x} \mid \mathbf{y})
$$

for all possible states $\mathbf{x}$, where $P(\mathbf{x} \mid \mathbf{y})$ denotes the posterior distribution of the state $\mathbf{x}$ given the data $\mathbf{y}$. That is, the state $\hat{\mathbf{x}}$ in (7) is the posterior mode.

If the random vectors in both (1) and (2) are Gaussian then, up to an additive constant, $-2 \log P(\mathbf{x} \mid \mathbf{y})$ is equal to

$$
(\mathbf{y}-\mathbf{F}(\mathbf{x}))^{\prime} \mathbf{S}_{\varepsilon}^{-1}(\mathbf{y}-\mathbf{F}(\mathbf{x}))+\left(\mathbf{x}-\mathbf{x}_{\alpha}\right)^{\prime} \mathbf{S}_{\alpha}^{-1}\left(\mathbf{x}-\mathbf{x}_{\alpha}\right)
$$

and finding the posterior mode is equivalent to minimizing (8) with respect to $\mathbf{x}$. If the random vectors $\varepsilon$ in (1) and $\alpha$ in (2) are more generically distributed according to Dist, the criterion (8) can still be used, since it solves the general problem of regularization formulated independently by Tikhonov [1963] and Twomey [1963].

Define the Jacobian,

$$
\mathbf{K}(\mathbf{x}) \equiv \frac{\partial \mathbf{F}(\mathbf{x})}{\partial \mathbf{x}}
$$

which is a matrix of order $n_{\varepsilon} \times n_{\alpha}$. Minimizing expression (8) implies that $\mathbf{x}$ satisfies

$$
-\mathbf{K}(\mathbf{x})^{\prime} \mathbf{S}_{\varepsilon}^{-1}(\mathbf{y}-\mathbf{F}(\mathbf{x}))+\mathbf{S}_{\alpha}^{-1}\left(\mathbf{x}-\mathbf{x}_{\alpha}\right)=\mathbf{0}
$$

which consists of $n_{\alpha}$ equations in $n_{\alpha}$ unknowns. The Gauss-Newton iteration scheme to solve (10) results in

$$
\mathbf{x}^{(\ell+1)}=\mathbf{x}^{(\ell)}+\left\{\mathbf{S}_{\alpha}^{-1}+\left(\mathbf{K}^{(\ell)}\right)^{\prime} \mathbf{S}_{\varepsilon}^{-1} \mathbf{K}^{(\ell)}\right\}^{-1}\left[\left(\mathbf{K}^{(\ell)}\right)^{\prime} \mathbf{S}_{\varepsilon}^{-1}\left(\mathbf{y}-\mathbf{F}\left(\mathbf{x}^{(\ell)}\right)\right)-\mathbf{S}_{\alpha}^{-1}\left(\mathbf{x}^{(\ell)}-\mathbf{x}_{\alpha}\right)\right]
$$

where $\mathbf{K}^{(\ell)} \equiv \mathbf{K}\left(\mathbf{x}^{(\ell)}\right)$ and $\ell=1,2, \ldots$ Equation (11) is iterated for increasing $\ell=1,2, \ldots$, until convergence, resulting in the estimated state vector, $\hat{\mathbf{x}}$. Now (11) can be unstable, so the OCO-2 algorithm uses a Levenberg-Marquardt approach [Levenberg, 1944; Marquardt, 1963] to solve (10).

By way of introduction to the next section, we would like to point out that the Jacobian matrix, $\mathbf{K}(\mathbf{x})$, is a first-order quantity, obtained from a first-order, multivariate Taylor series expansion; it is the first derivative of the forward model, $\mathbf{F}(\mathbf{x})$, and it captures the locally linear behavior of $\mathbf{F}$. However, if the Taylor series expansion is extended to second order, the second derivative of $\mathbf{F}(\mathbf{x})$, which is called the Hessian array $\left\{H_{i j k}(\mathbf{x})\right\}$, is needed to obtain a second-order approximation of the bias of the retrieval. Since the Hessian array is the second derivative of the $n_{\varepsilon}$-dimensional vector $\mathbf{F}(\mathbf{x})$, it is defined for $i=1, \ldots, n_{\varepsilon}, j=1, \ldots, n_{\alpha}$, and $k=1, \ldots, n_{\alpha}$. For the remote sensing application considered in section 5 , the number of entries in the Hessian array is $2040 \times 50 \times 50$.

\section{Bias and Mean Squared Prediction Error}

In general, the forward model is nonlinear, and a statistical error analysis of the optimally estimated state $\hat{\mathbf{x}}$ should recognize the consequences of this. In this section, the delta method [e.g., Meyer, 1975, chap 10] is used to obtain approximations for the bias vector, $E(\hat{\mathbf{x}}-\mathbf{x})$, and the mean square prediction error (MSPE) matrix, $E\left\{(\hat{\mathbf{x}}-\mathbf{x})(\hat{\mathbf{x}}-\mathbf{x})^{\prime}\right\}$. Nonlinearity in the forward model leads to nonzero bias of $\hat{\mathbf{x}}$, which the OE error analysis of Rodgers [2000] neglects by assuming that it is zero. 
The converged solution to (11) (or the Levenberg-Marquardt version of this) satisfies

$$
\begin{aligned}
\hat{\mathbf{x}} & =\mathbf{x}_{\alpha}+\mathbf{G}(\hat{\mathbf{x}})\left\{(\mathbf{y}-\mathbf{F}(\hat{\mathbf{x}}))+\mathbf{K}(\hat{\mathbf{x}})\left(\hat{\mathbf{x}}-\mathbf{x}_{\alpha}\right)\right\} \\
& =\mathbf{x}_{\alpha}+\mathbf{G}(\hat{\mathbf{x}})(\mathbf{F}(\mathbf{x})-\mathbf{F}(\hat{\mathbf{x}}))+\mathbf{A}(\hat{\mathbf{x}})\left(\hat{\mathbf{x}}-\mathbf{x}_{\alpha}\right)+\mathbf{G}(\hat{\mathbf{x}}) \varepsilon
\end{aligned}
$$

where

$$
\mathbf{G}(\mathbf{x}) \equiv\left\{\mathbf{S}_{\alpha}^{-1}+\mathbf{K}(\mathbf{x})^{\prime} \mathbf{S}_{\varepsilon}^{-1} \mathbf{K}(\mathbf{x})\right\}^{-1} \mathbf{K}(\mathbf{x})^{\prime} \mathbf{S}_{\varepsilon}^{-1}
$$

and

$$
\mathbf{A}(\mathbf{x}) \equiv \mathbf{G}(\mathbf{x}) \mathbf{K}(\mathbf{x})
$$

The quantities $\mathbf{G}(\mathbf{x})$ and $\mathbf{A}(\mathbf{x})$ are called the gain matrix and the averaging-kernel matrix, respectively, and in general, they are functions of the true state $\mathbf{x}$.

4.1. MSPE Matrix

Cressie and Wang [2013] use the delta method in the multivariate setting to show that to second order

$$
\begin{aligned}
\operatorname{cov}(\hat{\mathbf{x}}) \simeq & \tilde{\operatorname{cov}}(\hat{\mathbf{x}}) \equiv \mathbf{A}\left(\mathbf{x}_{\alpha}\right) \mathbf{S}_{\alpha} \mathbf{A}\left(\mathbf{x}_{\alpha}\right)^{\prime}+\mathbf{G}\left(\mathbf{x}_{\alpha}\right) \mathbf{S}_{\varepsilon} \mathbf{G}\left(\mathbf{x}_{\alpha}\right)^{\prime} \\
& \operatorname{cov}(\hat{\mathbf{x}}, \mathbf{x}) \simeq \widetilde{\operatorname{cov}}(\hat{\mathbf{x}}, \mathbf{x}) \equiv \mathbf{A}\left(\mathbf{x}_{\alpha}\right) \mathbf{S}_{\alpha},
\end{aligned}
$$

and

$$
\operatorname{cov}(\hat{\mathbf{x}}, \varepsilon) \simeq \widetilde{\operatorname{cov}}(\hat{\mathbf{x}}, \varepsilon) \equiv \mathbf{G}\left(\mathbf{x}_{\alpha}\right) \mathbf{S}_{\varepsilon}
$$

When the forward model $\mathbf{F}()$ is linear, $\mathbf{A}\left(\mathbf{x}_{\alpha}\right)$ and $\mathbf{G}\left(\mathbf{x}_{\alpha}\right)$ do not depend on $\mathbf{x}_{\alpha}$, and " $\simeq$ " becomes " $=$ " in the three relations above.

The delta method in statistics involves a Taylor series expansion, usually in a univariate setting, but here for a vector. We give the expansion of the retrieval $\hat{\mathbf{x}}$ about the prior mean $\mathbf{x}_{\alpha}$, although our results are true for the expansion about any fixed vector $\mathbf{x}^{0}$. If the prior mean $\mathbf{x}_{\alpha}$ is believed to be less reliable due to insufficient knowledge of the statistical structure of the true state, a better choice for the Taylor series expansion might be a starting vector of the iteration that solves (10). (We note that expanding the Taylor series about the retrieved vector $\hat{\mathbf{x}}$ confounds the variability due to nonlinearity with the variability due to the retrieval. It also raises more questions than it resolves, since if there is doubt about $\mathbf{x}_{\alpha}$ being a good prior mean, there is a consequent bias in $\hat{\mathbf{x}}$, as well as the nonlinearity bias established by our research. Many implementations of $\mathrm{OE}$, in fact, expand about $\hat{\mathbf{x}}$ [see O'Dell et al., 2012]).

To second order, the MSPE matrix can be approximated as follows: $E\left\{(\hat{\mathbf{x}}-\mathbf{x})(\hat{\mathbf{x}}-\mathbf{x})^{\prime}\right\} \simeq \widetilde{\mathbf{M S P E}}\left(\mathbf{x}_{\alpha}\right)$, where

$$
\tilde{\operatorname{MSPE}}\left(\mathbf{x}_{\alpha}\right) \equiv\left(\mathbf{A}\left(\mathbf{x}_{\alpha}\right)-\mathbf{I}\right) \mathbf{S}_{\alpha}\left(\mathbf{A}\left(\mathbf{x}_{\alpha}\right)-\mathbf{I}\right)^{\prime}+\mathbf{G}\left(\mathbf{x}_{\alpha}\right) \mathbf{S}_{\varepsilon} \mathbf{G}\left(\mathbf{x}_{\alpha}\right)^{\prime},
$$

which is derived in Cressie and Wang [2013]. Once again, when the forward model $\mathbf{F}()$ is linear, $\mathbf{A}\left(\mathbf{x}_{\alpha}\right)$ and $\mathbf{G}\left(\mathbf{x}_{\alpha}\right)$ do not depend on $\mathbf{x}_{\alpha}$ ' $\simeq$ " becomes " $=$," and the MSPE matrix is exactly equal to (14). Using standard matrix algebra, it is straightforward to show that (14) is equivalent to

$$
\left(\mathbf{K}(\mathbf{x})^{\prime} \mathbf{S}_{\varepsilon}^{-1} \mathbf{K}(\mathbf{x})+\mathbf{S}_{\alpha}^{-1}\right)^{-1} \equiv \hat{\mathbf{S}}
$$

which OCO-2 calls the error covariance matrix [O'Dell et al., 2012; Crisp et al., 2014]. Strictly speaking, $\hat{\mathbf{S}}$ should be called the mean square prediction error matrix; it is easy to see that $\hat{\mathbf{S}}$ is the error covariance matrix only when the bias vector is zero.

\subsection{Bias Vector}

Because of the nonlinearity of the forward model, $\hat{\mathbf{x}}$ will be a biased estimate of the state vector $\mathbf{x}$. That is, the retrieval error, $\hat{\mathbf{x}}-\mathbf{x}$, will have a distribution that is offset from $\mathbf{0}$. To obtain the bias, $E(\hat{\mathbf{x}}-\mathbf{x})$, one can again use the delta method by expanding the mean of $\hat{\mathbf{x}}$ around its prior mean $\mathbf{x}_{\alpha}$ and keeping terms of the expansion up to second order. Then, to second order, the bias vector can be approximated as follows: $E(\hat{\mathbf{x}}-\mathbf{x}) \simeq \tilde{\operatorname{bias}}\left(\mathbf{x}_{\alpha}\right)$, where 


$$
\begin{aligned}
& \tilde{\operatorname{bias}}\left(\mathbf{x}_{\alpha}\right) \equiv(1 / 2)\left(\begin{array}{c}
\left(\operatorname{vec}\left(\frac{\partial \mathbf{A}\left(\mathbf{x}_{\alpha}\right)_{1 \text { st row }}}{\partial \mathbf{x}_{\alpha}}\right)\right)^{\prime} \\
\left(\operatorname{vec}\left(\frac{\partial \mathbf{A}\left(\mathbf{x}_{\alpha}\right)_{\text {nnd row }}}{\partial \mathbf{x}_{\alpha}}\right)\right)^{\prime} \\
\vdots \\
\left(\operatorname{vec}\left(\frac{\partial \mathbf{A}\left(\mathbf{x}_{\alpha}\right)_{n_{\alpha} \text { th row }}}{\partial \mathbf{x}_{\alpha}}\right)\right)^{\prime}
\end{array}\right)^{\prime} \cdot \operatorname{vec}\left(\tilde{\mathbf{M}} \mathbf{S P E}\left(\mathbf{x}_{\alpha}\right)+2 \mathbf{S}_{\alpha} \mathbf{A}\left(\mathbf{x}_{\alpha}\right)^{\prime}\right) \\
&-(1 / 2)\left(\begin{array}{l}
\left(\operatorname{vec}\left(\frac{\partial \mathbf{G}\left(\mathbf{x}_{\alpha}\right)_{1 \text { st row }}}{\partial \mathbf{x}_{\alpha}}\right)\right)^{\prime} \\
\left(\operatorname{vec}\left(\frac{\partial \mathbf{G}\left(\mathbf{x}_{\alpha}\right)_{\text {nnd row }}}{\partial \mathbf{x}_{\alpha}}\right)\right)^{\prime} \\
\vdots \\
\left(\operatorname{vec}\left(\frac{\partial \mathbf{G}\left(\mathbf{x}_{\alpha}\right)_{n_{\alpha} \text { th row }}}{\partial \mathbf{x}_{\alpha}}\right)\right)^{\prime}
\end{array}\right) \cdot \operatorname{vec}\left(\mathbf{K}\left(\mathbf{x}_{\alpha}\right) \cdot \tilde{\mathbf{M}} \mathbf{S P E}\left(\mathbf{x}_{\alpha}\right)-2 \mathbf{S}_{\varepsilon} \mathbf{G}\left(\mathbf{x}_{\alpha}\right)^{\prime}\right)
\end{aligned}
$$

which is derived in Cressie and Wang [2013]. In (15), the "vec" operator is defined as follows: For any $m \times n$ matrix $\mathbf{B} \equiv\left(\mathbf{B}_{1}, \cdots, \mathbf{B}_{m}\right)$ with $n$-dimensional columns $\mathbf{B}_{1}, \cdots, \mathbf{B}_{m}, \operatorname{vec}(\mathbf{B})$ is defined as the $m n$-dimensional vector, $\operatorname{vec}(\mathbf{B}) \equiv\left(\mathbf{B}_{1}^{\prime}, \cdots, \mathbf{B}_{m}^{\prime}\right)^{\prime}$. We call $\tilde{\mathbf{b} i a s}\left(\mathbf{x}_{\alpha}\right)$, defined by (15), the nonlinearity bias. Notice from (15) that this depends only on the parameters of the state space model; since the bias is the statistical expectation of the retrieval error, it does not depend on the data.

A consequence of the forward model $\mathbf{F}(\mathbf{x})$ being nonlinear is that $\mathbf{K}(\mathbf{x}), \mathbf{G}(\mathbf{x})$, and $\mathbf{A}(\mathbf{x})$ depend on the true state $\mathbf{x}$, and hence, their derivatives with respect to $\mathbf{x}$ are generally nonzero. When the forward model is linear, $\mathbf{K}, \mathbf{G}$, and $\mathbf{A}$ are constant, and hence, $E(\hat{\mathbf{x}}-\mathbf{x})=\mathbf{0}=\widetilde{\mathbf{b}} \mathbf{b a s}\left(\mathbf{x}_{\alpha}\right)$. In the next section, we see that a nonzero value for expression (15) is directly attributable to the Hessian array, which recall is the second derivative of the forward model.

\subsection{The Hessian}

In the bias formula (15), the partial derivatives, $\frac{\partial \mathbf{G}(\mathbf{x})}{\partial x_{k}}$ and $\frac{\partial \mathbf{A}(\mathbf{x})}{\partial x_{k}}$, are functions of the derivatives of the Jacobian, $\left\{\frac{\partial \mathbf{K}(\mathbf{x})}{\partial x_{k}}: k=1, \cdots, n_{\alpha}\right\}$; see the expressions just below (12). Specifically,

$$
\begin{aligned}
\frac{\partial \mathbf{G}(\mathbf{x})}{\partial x_{k}}= & -\left\{\mathbf{S}_{\alpha}^{-1}+\mathbf{K}(\mathbf{x})^{\prime} \mathbf{S}_{\varepsilon}^{-1} \mathbf{K}(\mathbf{x})\right\}^{-1}\left[\frac{\partial \mathbf{K}(\mathbf{x})^{\prime}}{\partial x_{k}} \mathbf{S}_{\varepsilon}^{-1} \mathbf{K}(\mathbf{x})+\mathbf{K}(\mathbf{x})^{\prime} \mathbf{S}_{\varepsilon}^{-1} \frac{\partial \mathbf{K}(\mathbf{x})}{\partial x_{k}}\right] \\
& \times\left\{\mathbf{S}_{\alpha}^{-1}+\mathbf{K}(\mathbf{x})^{\prime} \mathbf{S}_{\varepsilon}^{-1} \mathbf{K}(\mathbf{x})\right\}^{-1} \mathbf{K}(\mathbf{x})^{\prime} \mathbf{S}_{\varepsilon}^{-1} \\
& +\left\{\mathbf{S}_{\alpha}^{-1}+\mathbf{K}(\mathbf{x})^{\prime} \mathbf{S}_{\varepsilon}^{-1} \mathbf{K}(\mathbf{x})\right\}^{-1} \frac{\partial \mathbf{K}(\mathbf{x})^{\prime}}{\partial x_{k}} \mathbf{S}_{\varepsilon}^{-1},
\end{aligned}
$$

and

$$
\frac{\partial \mathbf{A}(\mathbf{x})}{\partial x_{k}}=\frac{\partial \mathbf{G}(\mathbf{x})}{\partial x_{k}} \mathbf{K}(\mathbf{x})+\mathbf{G}(\mathbf{x}) \frac{\partial \mathbf{K}(\mathbf{x})}{\partial x_{k}}
$$

Thus, calculation of the partial derivatives of the Jacobian, $\left\{\frac{\partial \mathbf{K}(\mathbf{x})}{\partial x_{k}}: k=1, \ldots, n_{\alpha}\right\}$, is needed for calculating the nonlinearity bias given by (15). Notice that because $\mathbf{K}(\mathbf{x})$ is an $n_{\varepsilon} \times n_{\alpha}$ matrix, the array just above has $n_{\varepsilon} \times n_{\alpha} \times n_{\alpha}$ elements.

Now, since the Jacobian is itself a first derivative, any element in the array can be written as

$$
H_{i j k}(\mathbf{x}) \equiv \frac{\partial^{2} F_{i}(\mathbf{x})}{\partial x_{j} \partial x_{k}} ; i=1, \ldots, n_{\varepsilon}, j=1, \ldots, n_{\alpha}, k=1, \ldots, n_{\alpha}
$$

We call $\left\{H_{i j k}(\mathbf{x})\right\}$ the Hessian array; the subscript $i$ ranges over $n_{\varepsilon}$ elements, which for the OCO-2 instrument come from three spectral bands (Oxygen A band, Weak $\mathrm{CO}_{2}$ band, and Strong $\mathrm{CO}_{2}$ band); the subscripts $j$ and $k$ each range over $n_{\alpha}$ elements.

Figure 2 illustrates the structure of the Hessian array. 


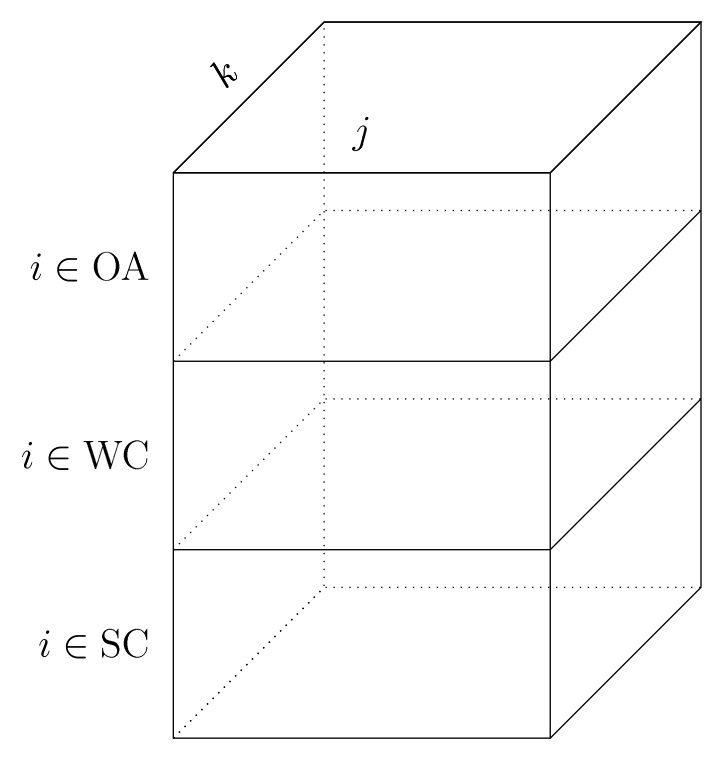

Figure 2. The form of the $n_{\varepsilon} \times n_{\alpha} \times n_{\alpha}$ Hessian array. For the OCO-2 algorithm, the index $i$ ranges over the Oxygen $A$ (OA) band, the Weak $\mathrm{CO}_{2}$ (WC) band, and the Strong $\mathrm{CO}_{2}$ (SC) band. For the retrievals in section $5, n_{\varepsilon} \times n_{\alpha} \times n_{\alpha}=$ $2040 \times 50 \times 50$; then the Hessian array has 5,100,000 entries.
An important property of the Hessian array (18) is that it is symmetric in $j$ and $k$. Thus, any estimate of it should preserve this property, namely, $H_{i j k}(\mathbf{x})=H_{i k j}(\mathbf{x})$. We achieve this below by using the approach of statistical estimating equations.

The Hessian element $H_{i j k}()$ can be estimated by taking a numerical derivative of the Jacobian, where the Jacobian is evaluated analytically: Let $K_{i j}(\mathbf{x})$ denote the $(i, j)$ th element of the $n_{\varepsilon} \times n_{\alpha}$ Jacobian matrix $\mathbf{K}(\mathbf{x})$ and define

$$
\tilde{H}_{i j k}(\mathbf{x}) \equiv \frac{K_{i j}\left(\mathbf{x}+\Delta_{k} \mathbf{e}_{k}\right)-K_{i j}(\mathbf{x})}{\Delta_{k}}
$$

for $i=1, \ldots, n_{\varepsilon}$ and $j, k=1, \ldots, n_{\alpha}$. In the expression (19), $\mathbf{e}_{k}$ is the vector with 1 as the $k$ th element and 0 everywhere else, and $\Delta_{k}>0$ is a small increment. In general, symmetry of the estimate (19) does not hold; that is, $\tilde{H}_{i j k}() \neq \tilde{H}_{i k j}()$.

The following approach to estimating $\left\{H_{i j k}(\mathbf{x})\right\}$ uses statistical estimating equations, and it will guarantee symmetry in $j$ and $k$ : There are two estimating equations for $H_{i j k}(\mathbf{x})$ that follow from (19), namely,

$$
\Delta_{k} H_{i j k}(\mathbf{x})=K_{i j}\left(\mathbf{x}+\Delta_{k} \mathbf{e}_{k}\right)-K_{i j}(\mathbf{x}),
$$

and

$$
\Delta_{j} H_{i j k}(\mathbf{x})=K_{i k}\left(\mathbf{x}+\Delta_{j} \mathbf{e}_{j}\right)-K_{i k}(\mathbf{x}) .
$$

Upon adding these two equations, we obtain

$$
\left(\Delta_{k}+\Delta_{j}\right) H_{i j k}(\mathbf{x})=K_{i j}\left(\mathbf{x}+\Delta_{k} \mathbf{e}_{k}\right)-K_{i j}(\mathbf{x})+K_{i k}\left(\mathbf{x}+\Delta_{j} \mathbf{e}_{j}\right)-K_{i k}(\mathbf{x}),
$$

which yields the estimate

$$
\hat{H}_{i j k}(\mathbf{x}) \equiv \tilde{H}_{i j k}(\mathbf{x})\left(\frac{\Delta_{k}}{\Delta_{j}+\Delta_{k}}\right)+\tilde{H}_{i k j}(\mathbf{x})\left(\frac{\Delta_{j}}{\Delta_{j}+\Delta_{k}}\right)
$$

for $i=1, \ldots, n_{\varepsilon}$ and $j, k=1, \ldots, n_{\alpha}$. Notice that the Hessian estimate (20) is a weighted combination of the asymmetric estimates given by (19), and it is easy to see that $\hat{H}_{i j k}(\mathbf{x}) \equiv \hat{H}_{i k j}(\mathbf{x})$, which is the required symmetry property in $j$ and $k$.

Now $\left\{\hat{H}_{i j k}(\mathbf{x})\right\}$ given by (20) is an estimate of $\left\{\frac{\partial \mathbf{K}(\mathbf{x})}{\partial x_{k}}: k=1, \ldots, n_{\alpha}\right\}$ which, from the formulas given at the beginning of this subsection, can then be used to obtain estimates of $\frac{\partial \mathbf{G}(\mathbf{x})}{\partial x_{k}}$ in (16) and $\frac{\partial \mathbf{A}(\mathbf{x})}{\partial x_{k}}$ in (17). Finally, then, the nonlinearity bias can be calculated from (15).

\section{Properties of the Retrieval Error of $\mathrm{XCO}_{2}$}

Section 2 describes an experiment we conducted to determine the influence of nonlinearity on the first two moments of the retrieval error. Our experiment exercises "strong control." This means that the functional form of the model and every parameter used to simulate forward from state mean $\mathbf{x}_{\alpha}$, to obtain true state $\mathbf{x}$ based on state covariance matrix $\mathbf{S}_{\alpha}$, and to finally obtain radiances $\mathbf{y}$ based on forward model $\mathbf{F}(\mathbf{x})$ and 
measurement-error covariance matrix $\mathbf{S}_{\varepsilon}$, are exactly the same when using the Levenberg-Marquardt algorithm to obtain the retrieval $\hat{\mathbf{x}}$. In this simulation experiment, states and measurements are realistic, and uncertainties are uniquely due to the ill-posed nature of retrieval and not due to using a misspecified forward function in the Levenberg-Marquardt algorithm.

Although $\mathbf{x}$ is known in the experiment, it is not used in the retrieval; its role is to allow us to determine the true retrieval error, $\hat{\mathbf{x}}-\mathbf{x}$. If the simulation is repeated $L$ times, where $L$ is large, then in obvious notation,

$$
\left\{\hat{\mathbf{x}}^{(l)}-\mathbf{x}^{(l)}: l=1, \ldots, L\right\}
$$

gives the distribution of the true retrieval error. We use $L=700$ below, for which relative Monte Carlo accuracy to the first decimal place is achieved.

Since the forward model $\mathbf{F}(\mathbf{x})$ is nonlinear, the statistical distribution of $\hat{\mathbf{x}}-\mathbf{x}$ is not Gaussian, although its first two moments remain important for inference. It is here where an MCMC approach would be a useful research tool, since the full posterior distribution is obtained. However, the excessive time taken to run the MCMC algorithm for a single retrieval (on the order of a day of CPU time; see section 1) means it is unlikely to become an operational tool.

The methodology proposed in this article is computationally efficient enough to be made operational on selected retrievals (e.g., when aerosols are present or for selected retrieval modes). The first moment expresses the bias of the retrieval,

$$
\text { bias } \equiv E(\hat{\mathbf{x}}-\mathbf{x})
$$

Inference on $\mathbf{x}$ from the estimate $\hat{\mathbf{x}}$ is based on the second central moment,

$$
\operatorname{cov} \equiv \operatorname{cov}(\hat{\mathbf{x}}-\mathbf{x})
$$

Notice that OCO-2's retrieval algorithm produces instead the second noncentral moment,

$$
\text { MSPE } \equiv E\left((\hat{\mathbf{x}}-\mathbf{x})(\hat{\mathbf{x}}-\mathbf{x})^{\prime}\right)
$$

which is appropriate when $\mathbf{b i a s}=\mathbf{0}$. In general,

$$
\operatorname{cov}=\text { MSPE }-(\text { bias })(\text { bias })^{\prime},
$$

and so cov can be recovered from the moments (22) and (24). In what follows in the experiment, we shall use (22) and (24) to summarize the results; these are sometimes called figures of merit [Cressie and Burden, 2015].

Apart from choosing 18 different location/seasons to generate a variety of atmospheric states, the two factors that we controlled for were the presence/absence of aerosols in the forward model (and hence in the retrieval), and the presence/absence of the nonlinearity bias correction. The "response" of the experiment centered on the first two moments of $X \mathrm{CO}_{2}$, the column-averaged $\mathrm{CO}_{2}$ dry air mole function. The state variable $X \mathrm{CO}_{2}$ is obtained by an appropriately weighted combination of the $n_{p}=20 \mathrm{VMRs}$ of $\mathrm{CO}_{2}$ at the 20 pressure levels given in ACOS/OCO-2's forward model [Crisp et al., 2014].

In what follows in this section, we write $x \equiv$ true $X \mathrm{CO}_{2}$ value at a given sounding, which in practice is an unknown scalar. Correspondingly, we write $\hat{x}(\mathbf{y}) \equiv$ estimated $X \mathrm{CO}_{2}$ value, featuring the radiances $\mathbf{y}$ measured at the given sounding. The theory of OE assumes that $x$ is a random variable, and from the theory of statistical estimation, $\hat{x}(\mathbf{y})$ is also a random variable with strong statistical dependence on $x$. Then the retrieval error for estimating $\mathrm{XCO}_{2}$ is

$$
\hat{x}(\mathbf{y})-x
$$

which is a random variable whose distribution is ultimately determined by the joint distribution of $x$ and $\mathbf{y}$.

Recall that there are $j=1, \ldots, 18$ diverse location/seasons and $I=1, \ldots, 700$ simulations for each location/ season, which we write as

$$
\left\{\hat{x}\left(y_{j}^{(l)}\right)-x_{j}^{(l)}: I=1, \ldots, 700\right\} ; j=1, \ldots, 18
$$


(a)

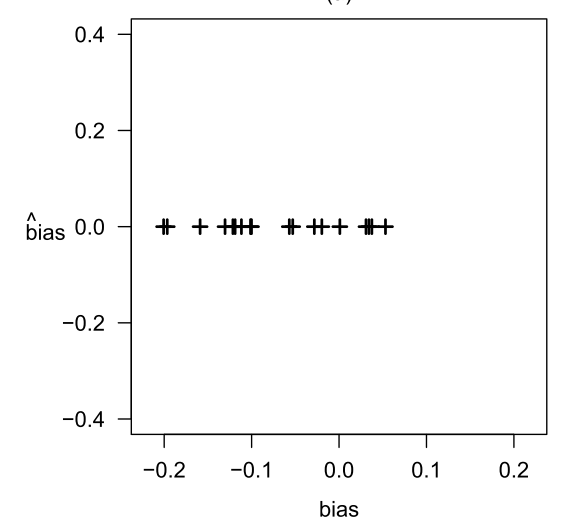

(b)

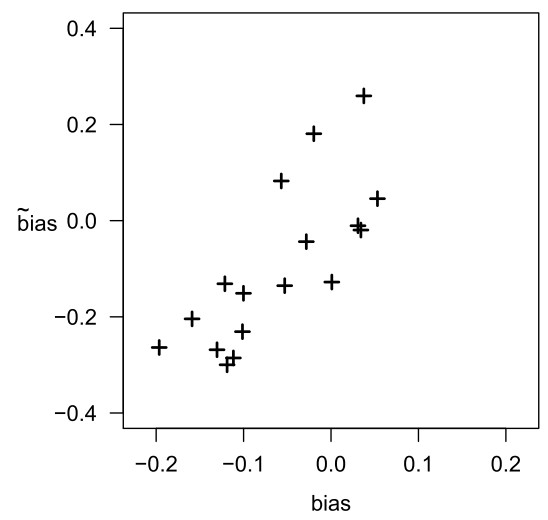

Figure 3. In the presence of aerosols, model-based biases are compared to the true bias. (a) For ACOS/OCO-2's error analysis, which is based on a linear approximation, $\hat{b i a s}=0$; see (28). (b) For the nonlinear error analysis based on the delta method given in section 4 , bias is given by (27). The horizontal axes in both plots show the true biases obtained from the first moment of the estimation error; see (25).

At the $j=1, \ldots, 18$ location/seasons (section 2), we define the bias

$$
\operatorname{bias}_{j} \equiv \sum_{l=1}^{700}\left(\hat{x}\left(y_{j}^{(l)}\right)-x_{j}^{(l)}\right) / 700,
$$

and the root-mean-square prediction error,

$$
\operatorname{rmspe}_{j} \equiv\left\{\sum_{l=1}^{700}\left(\hat{x}\left(y_{j}^{(l)}\right)-x_{j}^{(l)}\right)^{2} / 700\right\}^{1 / 2} .
$$

These are the true biases and root-mean-square predictor errors, which we can compute at the 18 location/seasons chosen for this experiment.

As was made clear in section 4, for the full state vector there is a first-order result based on a linearization of the forward model, and there is a second-order result based on the delta method that gives (13) and (15). For $X \mathrm{CO}_{2}$, the nonlinear approximations to the bias and MSPE are easily obtained as follows: First, $X \mathrm{CO}_{2}=\mathbf{w}^{\prime} \mathbf{x}$ and $\hat{\mathrm{XCO}} 2=\mathbf{w}^{\prime} \hat{\mathbf{x}}$ for known weight vector $\mathbf{w}$. Then, using obvious notation, the second-order result is

$$
\tilde{\operatorname{bias}}\left(X \mathrm{CO}_{2}\right)=\mathbf{w}^{\prime} \tilde{\operatorname{bias}}\left(\mathbf{x}_{\alpha}\right) ; \quad \tilde{\operatorname{MSPE}}\left(X \mathrm{CO}_{2}\right)=\mathbf{w}^{\prime} \tilde{\mathbf{M S P E}}\left(\mathbf{x}_{\alpha}\right) \mathbf{w}
$$

From O'Dell et al. [2012] and Crisp et al. [2012], the ACOS/OCO-2 algorithm uses

$$
\hat{\operatorname{bias}}\left(X \mathrm{CO}_{2}\right)=0 ; \quad \hat{\operatorname{MSPE}}\left(X \mathrm{CO}_{2}\right)=\mathbf{w}^{\prime} \tilde{\mathbf{M S P E}}(\hat{\mathbf{x}}) \mathbf{w}=\mathbf{w}^{\prime} \hat{\mathbf{S}} \mathbf{w},
$$

which is a result of a linear approximation to the forward model. That is, in ACOS/OCO-2's error analysis, a zero bias is obtained, and the MSPE matrix is evaluated at the retrieved state $\hat{\mathbf{x}}$.

For each of the $j=1, \ldots, 18$ location/seasons, we can make a comparison of the true bias, namely, bias $_{j}$, with $\hat{b i a s}_{j}(=0)$ from OE given by (28), and $\tilde{b i a s}_{j}\left(\equiv \tilde{\mathrm{bias}}\left(X \mathrm{CO}_{2 j}\right)\right)$ from (27). We can also make a comparison of

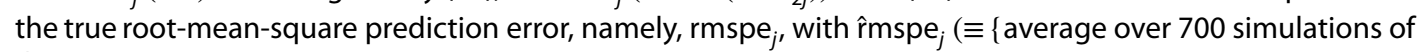
$\left.\hat{\operatorname{MSPE}}\left(X \mathrm{CO}_{2 j}\right)\right\}^{1 / 2}$ ) from OE given by (28). Finally, we can make a comparison of $\mathrm{rmspe}_{j}$ with $\tilde{r m s p e}_{j}\left(\equiv\left\{\tilde{M S P E}\left(X \mathrm{CO}_{2 j}\right)\right\}^{1 / 2}\right)$ from (27). These comparisons are made through $x$ - $y$ plots, each of which contains $18 x$-y pairs corresponding to the 18 location/seasons.

In Figures 3 and 4, we show only the results for the aerosols case. The clear-sky case (i.e., absence of aerosols) turns out to be less interesting because the nonlinearities in the forward model are much less pronounced. Aerosols confound the retrieval of $\mathrm{XCO}_{2}$ in a strongly nonlinear manner [O'Dell et al., 2012], so it is appropriate that our comparisons focus on this case. 
(a)

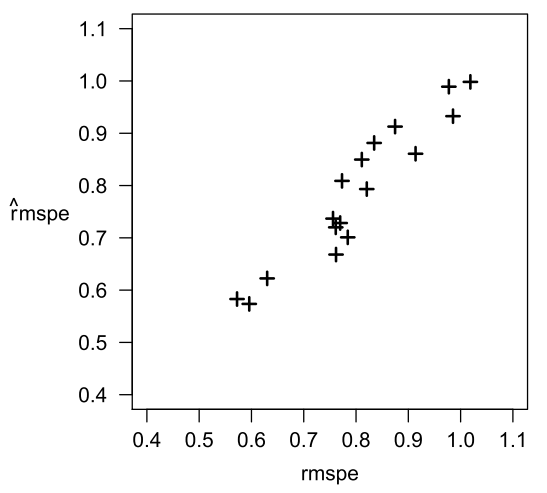

(b)

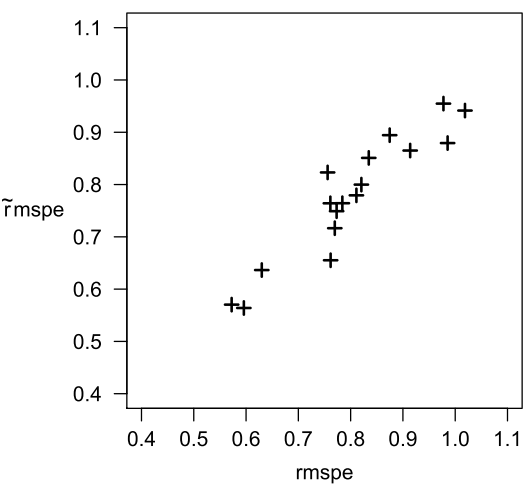

Figure 4. In the presence of aerosols, model-based root-mean-square prediction errors, $\hat{r}$ mspe, are given for ACOS/OCO-2's error analysis; (a) r̂mspe is obtained from averaging (28) over all 700 simulations and then taking the square root. The model-based root-mean-square prediction errors, rmspe, are given for the nonlinear error analysis based on the delta method; (b) rmspe is the same for all simulations and is obtained from (27). The horizontal axes in both plots show the true root-mean-square prediction errors obtained from (26).

By comparing Figure 3a with Figure 3b, one can see the benefit of accounting for the nonlinearity bias when the forward model has strong nonlinearities. Statistical theory indicates that there will be very little difference in the root-mean-square prediction errors, and this is confirmed when comparing Figures $4 \mathrm{a}$ and $4 \mathrm{~b}$.

In our experiment, we have chosen a realistic set of atmospheres with aerosols, and we have found that the true nonlinearity bias given by (25) is no more extreme than $\pm 0.2 \mathrm{ppm}$. Similar experiments conducted on an earlier version of the ACOS/OCO-2 algorithm (B2.8) showed much more serious biases, on the order of $\pm 1 \mathrm{ppm}$ and sometimes worse. The forward model that was behind the earlier versions was not effectively capturing the radiative transfer with more state elements (as many as 112) that were highly correlated. As a consequence, the inverse problem was more ill posed, and the retrieval error was more biased. Based on the approach we have taken to assessing retrieval error, we have been able to quantify an improvement in the ACOS/OCO-2 algorithm from B2.8 to B5.0.

\section{Discussion and Conclusions}

The OE approach to obtaining retrievals in remote sensing incorporates physical knowledge, applied mathematics, and statistics. It is akin to the well-known approach in the signal-processing literature called state space estimation, where both the estimated state and the true state are random, and the error analysis goes beyond a linear approximation [e.g., Shumway and Stoffer, 2006, chap 6]. In this article, we have shown how statistical theory (posterior analysis, delta method, and estimating equations) can be used to yield an uncertainty quantification (namely, the first two moments) of the retrieval error, by recognizing the nonlinearity of the forward model and all sources of randomness. The first two moments of the retrieval error are fundamental quantities used for $\mathrm{CO}_{2}$ flux inversions from remote sensing data.

The nonlinearity in the forward model results in a nonlinearity bias that is estimated using a weighted estimate of the Hessian array; this approximation improves as the signal-to-noise ratio increases [Cressie and Wang, 2013]. Using the ACOS/OCO-2 algorithm B5.0, $X \mathrm{CO}_{2}$ retrievals in a controlled experiment (section 2 ) exhibit absolute nonlinearity biases up to $0.2 \mathrm{ppm}$ when aerosols are present (section 5). For clear-sky retrievals, biases are effectively zero.

Of course, there are other sources of bias, some known for physical reasons and some described by regression relationships. Validation of the $\mathrm{XCO}_{2}$ product is based on data from the Total Carbon Column Observing Network (TCCON) [e.g., Wunch et al., 2011]. In fact, a TCCON "ground truth" datum is also an estimate of $X \mathrm{CO}_{2}$ derived from a different nonlinear forward model, and it has its own nonlinearity bias. Our results in section 5 indicate that under controlled conditions the nonlinearity bias of XCO2 retrievals is within the limits of the error characteristics of TCCON. In actual retrievals, bias can arise from a number of components; our results indicate that the nonlinearity component by itself would not be seen by an appropriate comparison to TCCON data. 


\section{Acknowledgments}

The research described in this article was performed for the Orbiting Carbon Observatory Project at the Jet Propulsion Laboratory, California Institute of Technology, under contract with the National Aeronautics and Space Administration (NASA). Cressie's and Wang's research was supported by NASA grant NNH11-ZDA001N-OCO2. Data are available from Mike Smyth, JPL (mike.m.smyth@jpl.nasa.gov). The authors would like to thank A. Braverman, J. Brynjarsdóttir, R. Castano, A. Eldering, J. Hobbs, M. Gunson, D. O'Brien, C. O'Dell, and I. Polonsky for their comments on various presentations of this research. We would also like to thank C. Frankenberg for his assistance in setting up the realistic simulation experiment. B. Maloney and S. Burden provided invaluable assistance to the preparation of this manuscript. Finally, our sincere gratitude goes to two anonymous referees and the Editor whose comments were extremely helpful in revising our article.
The specification of a prior distribution (usually in the form of a prior mean vector and a prior covariance matrix) for the unknown state is required for OE. The robustness of the estimates and of the associated error analysis to misspecification of the prior has not been addressed in this article. Our goal has been to work within the assumptions of $\mathrm{OE}$ to obtain $\mathrm{XCO}_{2}$ estimates and their uncertainty quantification that accounts for the nonlinearity in the forward model.

\section{References}

Böesch, H., et al. (2006), Space-based near-infrared $\mathrm{CO}_{2}$ measurements: Testing the Orbiting Carbon Observatory retrieval algorithm and validation concept using SCIAMACHY observations over Park Falls, Wisconsin, J. Geophys. Res., 111, D23302, doi:10.1029/2006JD007080

Böesch, H., D. Baker, B. Connor, D. Crisp, and C. Miller (2011), Global characterization of $\mathrm{CO}_{2}$ column retrievals from shortwave-infrared satellite observations of the Orbiting Carbon Observatory-2 mission, Remote Sens., 3, 270-304.

Bréon, F.-M., and P. Ciais (2010), Spaceborne remote sensing of greenhouse gas concentrations, C. R. Geosci., 342, $412-424$.

Connor, B. J., H. Böesch, G. Toon, B. Sen, C. Miller, and D. Crisp (2008), Orbiting carbon observatory: Inverse method and prospective error analysis, J. Geophys. Res, 113, D05305, doi:10.1029/2006JD008336.

Cressie, N., and S. Burden (2015), Figures of merit for simultaneous inference and comparisons in simulation experiments, Stat, 4, $196-211$. Cressie, N., and R. Wang (2013), Statistical properties of the state obtained by solving a nonlinear multivariate inverse problem, Appl. Stochastic Models Bus. Ind., 29, 424-438.

Crisp, D., et al. (2012), The ACOS $X_{\mathrm{CO}_{2}}$ retrieval algorithm. Part 2: Global $X_{\mathrm{CO}_{2}}$ data characterization, Atmos. Meas. Tech., 5, $687-707$. Crisp, D., et al. (2014), OCO (Orbiting Carbon Observatory)-2 Level 2 Full Physics Retrieval Algorithm Theoretical Basis, Pasadena, Calif. Kuze, A., H. Suto, M. Nakajima, and T. Hamazaki (2009), Thermal and near infrared sensor for carbon observation Fourier-transform spectrometer on the Greenhouse Gases Observing Satellite for greenhouse gases monitoring, Appl. Opt., 48, 6716-6733. Levenberg, K. (1944), A method for the solution of certain nonlinear problems in least squares, Q. J. Appl. Math., 2, $164-168$. Marquardt, D. W. (1963), An algorithm for least-squares estimation of nonlinear parameters, J. Soc. Ind. Appl. Math., 11, 431 -441. Meyer, S. L. (1975), Data Analysis for Scientists and Engineers, Wiley, New York.

O'Dell, C. W., et al. (2012), The ACOS $\mathrm{CO}_{2}$ retrieval algorithm — Part 1: Description and validation against synthetic observations, Atmospheric Measurement Techniques, 5, 99-121.

Rodgers, C. D. (2000), Inverse Methods for Atmospheric Sounding, World Sci., Singapore.

Shumway, R., and D. S. Stoffer (2006), Time Series Analysis and Its Applications, With R Examples, 2nd edn., Springer, New York.

Tamminen, J. (2004), Validation of nonlinear inverse algorithms with Markov chain Monte Carlo method, J. Geophys. Res., 109, D19303.

Tamminen, J., and E. Kyrola (2001), Bayesian solution for nonlinear and non-Gaussian inverse problems by Markov chain Monte Carlo method, J. Geophys. Res., 106, 14,377-14,390.

Tikhonov, A. N. (1963), On the solution of incorrectly stated problems and a method of regularization, Dokl. Acad. Nauk SSSR, 151, 501-504. Twomey, S. (1963), On the numerical solution of Fredholm integral equation of the first kind by the inversion of the linear system produced by quadrature, J. Assoc. Comput. Mach., 10, 97-101.

Wunch, D., et al. (2011), A method for evaluating bias in global measurements of $\mathrm{CO}_{2}$ total columns for space, Atmos. Chem. Phys., 11 , $12,317-12,337$ 